\title{
State transitions and jet formation in black hole binaries
}

\author{
Emrah Kalemci* \\ Sabancı University, Orhanlt- Tuzla, Istanbul, TURKEY \\ E-mail: ekalemciesabanciuniv.edu \\ John A. Tomsick \\ SSL, UC Berkeley \& CASS, UC San Diego, CA, USA. \\ E-mail: jtomsick@ssl.berkeley.edu

\section{Richard E. Rothschild, Katja Pottschmidt, Simone Migliari \\ CASS, UC San Diego, CA, USA.}

\section{Stephane Corbel}

CEA Saclay, Service d'Astrophysique, F-91191, Gif sur Yvette, France.

\section{Philip Kaaret}

Department of Physics and Astronomy, Un. of Iowa, IA, USA.

\begin{abstract}
The daily monitoring observations of black hole transients with RXTE provided important clues on the conditions of the state transitions, both in terms of spectra and timing. The recent addition of monitoring in the optical-infrared and the radio band significantly extended our knowledge of the relation between the jets and the spectral states. However, there are still very important unanswered questions, most importantly, whether the formation of the jet triggers any change in the spectral and temporal properties of the source. The answer to this question is also intrinsically related to the origin of the hard X-ray emission. In this work, the relation between the jet and the state transitions is discussed, using the data from GX 339-4, 4U 1543-47, H 1743-322, and GRO J1655-40, concentrating on the evolution of spectral and temporal parameters before, during and after the formation of the jet.
\end{abstract}

VI Microquasar Workshop: Microquasars and Beyond

September 18-22 2006

Società del Casino, Como, Italy

\footnotetext{
${ }^{*}$ Speaker.
} 


\section{Introduction}

\subsection{Spectral states}

The Galactic black hole transients (GBHTs) show several correlated spectral and temporal variability properties during outbursts. These "spectral states" were historically characterized using X-ray observations. We refer to McClintock \& Remillard 2006 [20] for detailed discussion of the spectral states based on the absolute X-ray spectral and temporal properties of GBHTs. We emphasize that properties in other wavelengths are also closely related to the spectral states of GBHTs. In the thermal dominant state (TDS), the radio emission from the compact core is often quenched [5]. Optically thin outflows are sometimes detected during state transitions [11], and powerful, compact jets are always observed in the low hard state (LHS) [10]. The optical and infrared (OIR) emission properties are also shown to depend on spectral states [18, 6, 25].

One can investigate the evolution during an outburst by determining the transitions, abrupt changes in spectral and temporal properties. An efficient way to do this is to plot "hardnessintensity" diagrams which show distinct areas of hardness and intensity separating spectral states $[13,1]$. The information that can be provided with hardness intensity diagrams is useful, especially to discuss the evolution during an entire outburst, but limited in the sense that only the evolution of a few variables can be shown. For a limited number of observations, one can also plot the evolution of several parameters as a function of time, and stack them to allow direct comparisons. In this case, one can also see the timescale of changes and determine the fast and slow transitions directly. This is the method we employ to investigate GBHTs during outburst decays.

\subsection{Black hole transients in outburst decays}

The multi-wavelength observations made during the outburst decays provide valuable information about GBHTs due to the transitions from the TDS to the hard-intermediate state (IS, [1]), and eventually to the low-hard state (LHS) [15]. Observing the changes during the transitions can reveal more information about the geometry and the physical environment of these systems [9, 32]. The LHS provides additional information with the variability properties, and strong radio and OIR emission, both correlating with spectral parameters. Our group has been observing these transients during outburst decay in X-rays with the Rossi X-ray Timing Explorer (RXTE), and in radio with five major motivations: (1) Characterizing the spectral and temporal evolution close to state transitions, (2) understanding the conditions for jet formation ${ }^{1}$, (3) quantifying the contribution of the jet to X-ray flux and spectrum, (4) looking for unprecedented phenomena, and (5) characterizing the spectral evolution towards quiescence [14, 27, 16, 29].

A uniform analysis of all black hole transients observed with $\sim$ daily coverage with $R X T E$ during outburst decay between 1996 and 2001 provided important information on the evolution of spectral and temporal parameters during the decay [17]. The sharpest change indicating a state transition is observed to be a change in the shape of the power spectral density (PSD), and a jump in the rms amplitude of variability from less than a few percent to more than tens of percent in less than a day (See Fig. 1). This change in the rms amplitude is always accompanied by a sharp increase in the power-law flux. There is also evidence that the strong rms noise is only observed

\footnotetext{
${ }^{1}$ In this work jet denotes the compact jet observed in the hard states of GBHTs, not the optically thin ejections.
} 


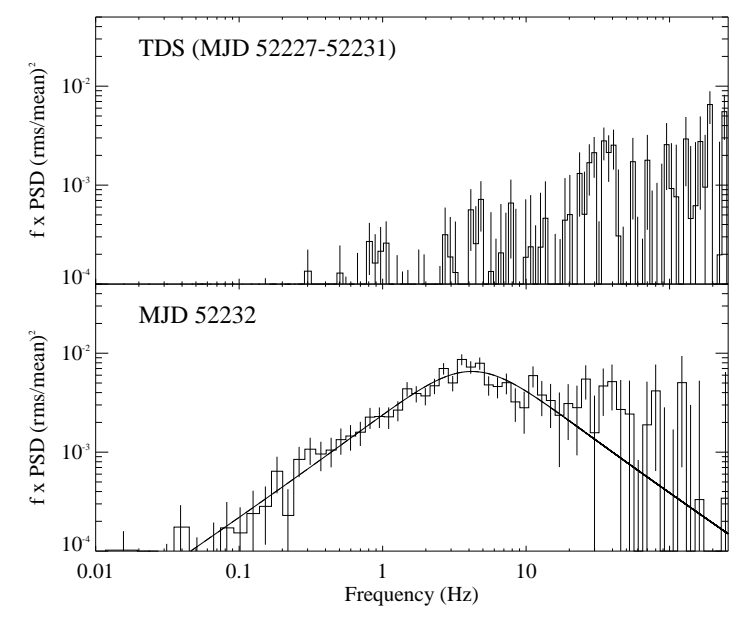

Figure 1: The transition in timing for XTE J1650-500. The time between the two PSDs is approximately one day [16].

when the ratio of the power-law flux to the total flux in 3-25 keV band (the power-law ratio, PLR) is above a certain percentage. During the outburst decay, the photon index, the disk temperature, and the disk flux usually decrease slowly. After the transition, characteristic frequencies of the power density spectrum also decrease with time.

\section{Analysis}

We apply a uniform analysis to all sources that we investigate. This allows us a direct comparison of source properties, and also to search for certain correlations between the spectral and temporal parameters. The analysis methods for the observations with RXTE are summarized below. In addition to the X-ray data, we obtain the radio, optical and infrared fluxes directly from the observer or from ATELs, and incorporate this information into our results.

\subsection{X-ray spectral analysis}

When possible, we use both the PCA and the HEXTE instruments on RXTE for the spectral analysis. For the PCA, the $3-25 \mathrm{keV}$ band is used. We add $0.8 \%$ up to $7 \mathrm{keV}$, and $0.4 \%$ above $7 \mathrm{keV}$ as systematic error. We use all available PCUs for each observation, choosing the combination that would give maximum number of counts per pointing. The $16-200 \mathrm{keV}$ band is used for the HEXTE data. The HEXTE background is measured throughout the observation by alternating between the source and background fields every $32 \mathrm{~s}$. The relative normalization between the PCA and the HEXTE is kept free. The Galactic ridge emission becomes a factor as the flux decreases. We use a combination of techniques to determine the ridge contribution; simultaneous observations of imaging instruments, Galactic Bulge Scans, quiescence state observations, or the literature [24,30].

For all the observations, our first spectral model consists of interstellar absorption, a smeared edge, a multicolor disk blackbody, a power law, and a narrow Gaussian to model the iron line, and the ridge emission model if necessary. This model has been commonly used for the spectral analysis of black holes in the LHS [26, 18]. Once we fit the observations with this model, we add a high energy cut-off to the model and do an F-test to determine if a cut-off is required. 


\subsection{X-ray temporal analysis}

For each observation, we compute the power density spectra (PDS) from the PCA data using IDL programs developed at the University of Tübingen [23]. The PDS is normalized as described in Miyamoto \& Kitamoto 1989 [22], and corrected for the dead-time effects according to Zhang et al, 1995 [33]. Using 256 second time segments, we investigate the low frequency QPOs and the timing properties of the continuum up to $256 \mathrm{~Hz}$ for different energy bands. We fit all our PDSs with broad and narrow Lorentzians with our standard timing analysis techniques [18, 15, 23].

The rms amplitudes are calculated over a frequency band from zero to infinity. We multiply the rms amplitude of variability with $\frac{T^{2}}{(T-(R+B))^{2}}$, where $T$ is the overall count rate, $B$ is the background rate determined using pcabackest, and $R$ is the count rate due to the Galactic ridge, to obtain the variability inherent to the source [3].

\section{Results}

In this work, we will concentrate on outburst decays of four sources. Results of our analysis from two of them, 4U 1543-47 and H 1743-322 were also published elsewhere [18, 19]. Preliminary results from GX 339-4 during its 2003 outburst decay and GRO J1655-40 during its 2005 outburst decay are shown for the first time in this work.

\section{$3.14 \mathrm{U} 1543-47$}

The details of the source, outburst and the multi-wavelength coverage can be found in [18]. Fig. 2 shows the evolution of some of the important spectral and temporal parameters of the system close to the state transitions during its decay. The plot indicates two state transitions: (1) There is a fast transition (timescale of one day) in timing marked (dashed line separating TD and IS) by a jump in the rms amplitude, which also coincides with a sudden increase in power-law flux. During this transition, the spectral index remains the same. There is no clear indication of radio or OIR activity. (2) There is a slower transition seen mainly in the spectral index as it decreases. The onset of this transition is marked by the second dashed line. It takes $\sim 7$ days for the spectral index to reach its hardest point. At the onset of this transition, a cut-off is required in the spectral fits. The OIR activity starts $\sim 4$ days after the onset of this second transition, and peaks when the spectral index is hardest. At the time OIR activity is seen, there is no need for a cut-off in the spectrum. While approaching quiescence, the spectra soften again.

\subsection{H $1743-322$}

The details of the source, outburst and the multi-wavelength coverage can be found in [19]. In Fig. 3 we show the evolution of major spectral and temporal parameters during the outburst decay. The results in terms of state transitions are similar to $4 \mathrm{U} 1543-47$, a fast transition in timing followed by a slower hardening of the spectral index. But there are also differences. There is no need for a high energy cut-off in any of the fits for this source. There is no clear sign of softening at the end of the outburst, though the error bars are too large to exclude this possibility.

Unlike other sources, this source shows a sudden disappearance of timing signature after it transited to the IS. On MJD 52933, for one observation, the power-law flux and the PLR dropped, 


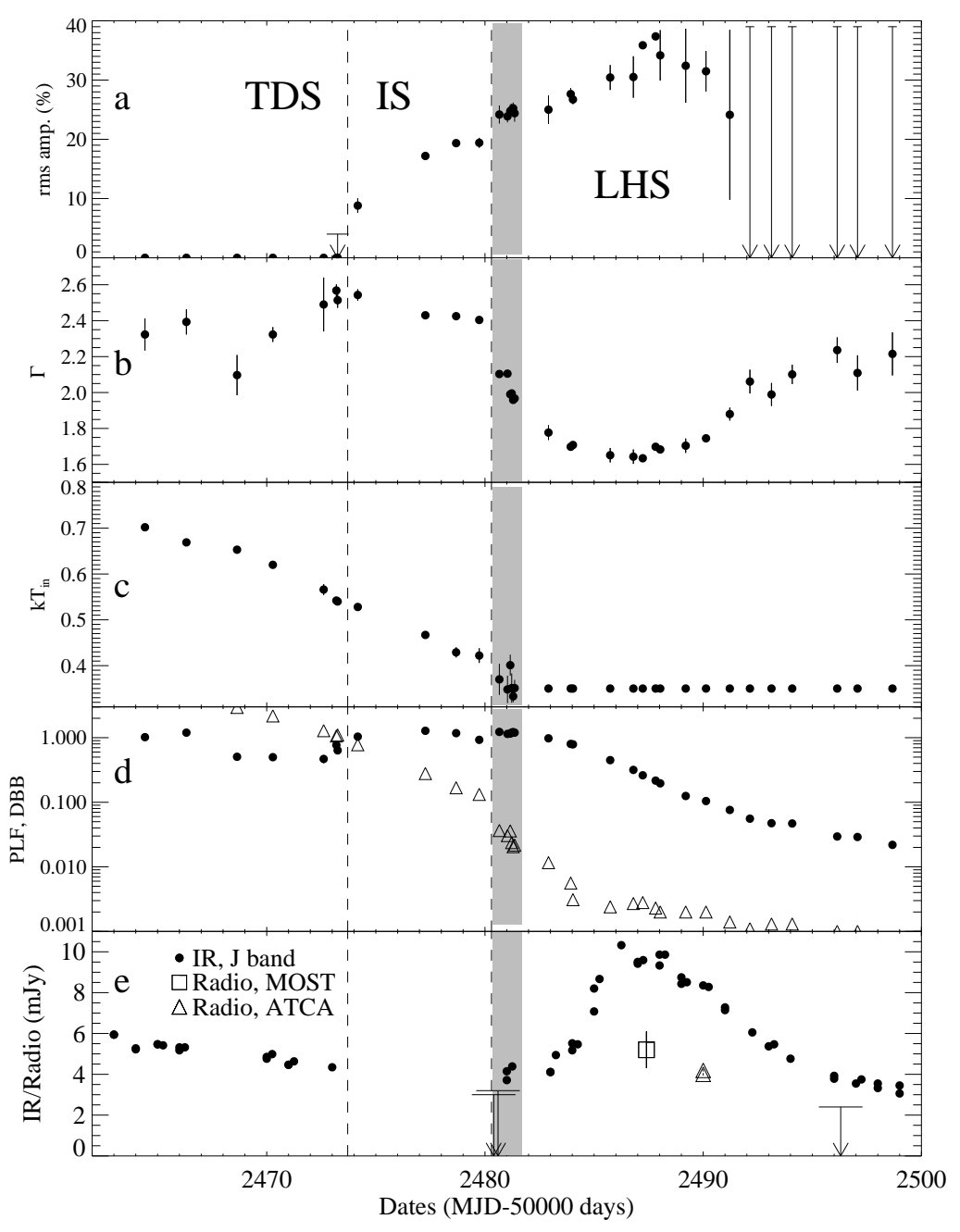

Figure 2: $4 \mathrm{U}$ 1543-47; the evolution of (a) the total rms amplitude of variability in 3-30 keV band, (b) the photon index $(\Gamma),(\mathrm{c})$ the inner disk temperature $\mathrm{k} T_{\text {in }}(\mathrm{d})$ unabsorbed power-law flux and the disk blackbody flux in 3-25 keV band in units of $10^{-9} \mathrm{ergs} \mathrm{cm}^{-2} \mathrm{~s}^{-1}$, (e) the J-band fluxes along with radio fluxes. The dashed lines indicate approximate times of state transitions. The gray area shows the observations during which a high energy cut-off is required in the fit.

and the PSD became similar to that of TDS (shown with red dots in Fig. 3). Based on the TDS observations and this one, we set a PLR threshold of variability of $\sim 0.6$ for $\mathrm{H} 1743-322$. We nicknamed this as "flip-flop" of states. This flip-flop undoubtedly links the power-law part of the spectrum to the variability.

\subsection{GX 339-4}

GX 339-4 is one of the recurrent GBHTs observed and analyzed intensively especially in the RXTE era. We analyzed the archival data from MJD 52680 to MJD 52785, and covered a region starting from the TDS to deep in the LHS during the decay. For GX 339-4, the ridge contribution is determined by spectral analysis of five observations in quiescence between the end of 2003 outburst 


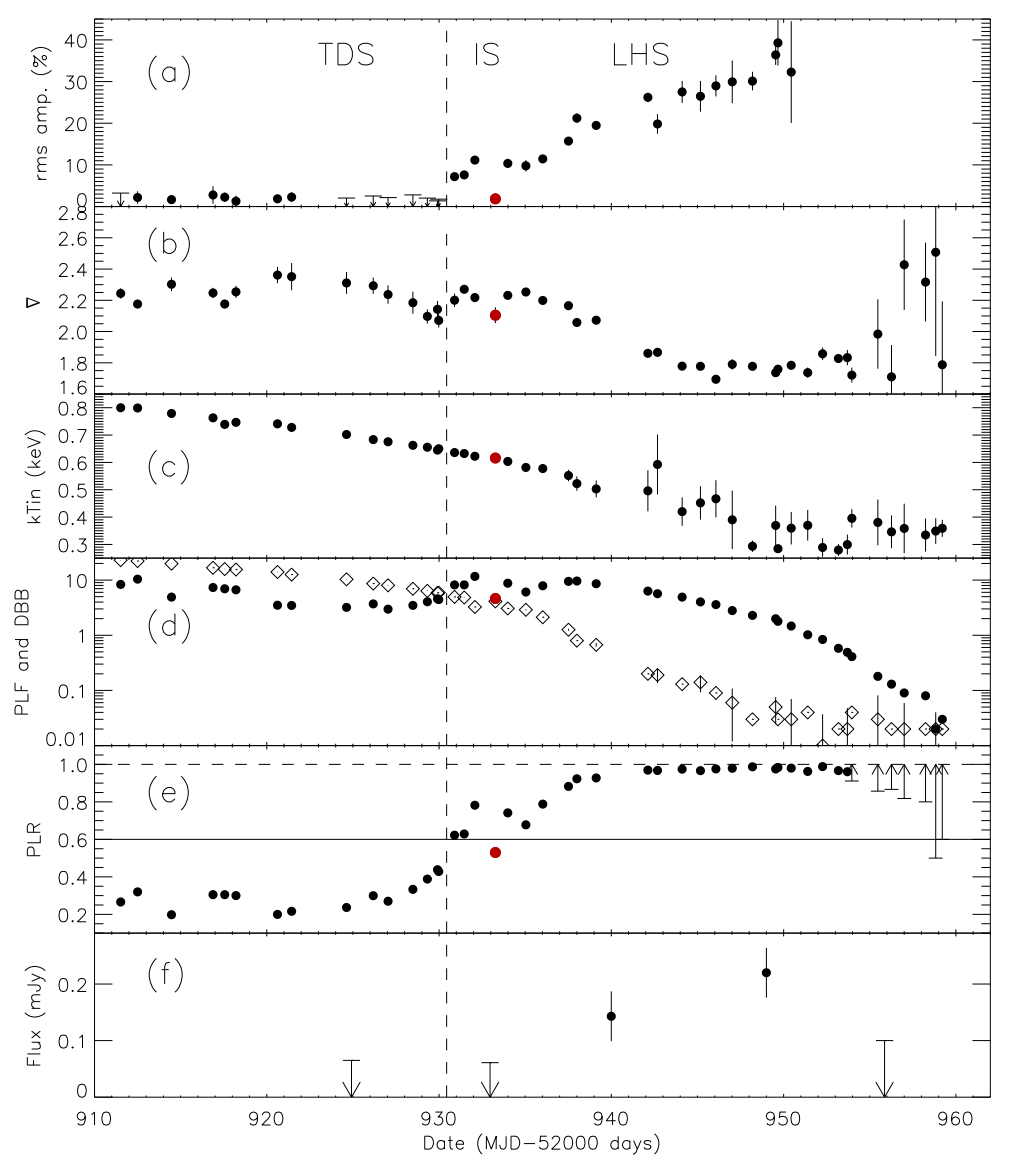

Figure 3: H 1743-322; the evolution of (a) the total rms amplitude of variability in 3-30 keV band, (b) the photon index $(\Gamma)$, (c) the inner disk temperature $\mathrm{k} T_{\text {in }}$ (d) unabsorbed power-law flux (filled circles) and the disk blackbody flux (triangles) in $3-25 \mathrm{keV}$ band in units of $10^{-9} \mathrm{ergs} \mathrm{cm}^{-2} \mathrm{~s}^{-1}$, (e) PLR, (f) the radio flux. The dashed line indicates approximate time of state transitions to the IS. The solid line in (e) indicates threshold to observe variability. Observation shown in red has reduced rms amplitude.

and the beginning of the 2005 outburst.

Fig. 4 summarizes the evolution of spectral and temporal parameters along with the evolution of the IR magnitudes. The fast and the slow transitions can be also seen in this source. Like $4 \mathrm{U} 1543-47$, during a limited time a high energy cut-off is required in the fit. The cut-off is required when the spectrum is hardest, and while the OIR flux is just rising. The rise of the OIR flux is after the onset of the slow transition. This source also shows a peculiar single observation. On MJD 52708, the C type QPO disappears, the rms amplitude of variability reduces significantly and a B type QPO appears (shown red in Fig. 4). This type of behavior (sudden appearance of B type QPO) during the rising phase of the outbursts usually coincide with radio ejections and sets the so-called "jet line" [1]. However, in this case, it is not associated with jet activity. At the end of the outburst, there may be a softening in the spectrum.

\subsection{GRO J1655-40}

GRO J1655-40 is another recurrent GBHT which had its most recent outburst in 2005. The 


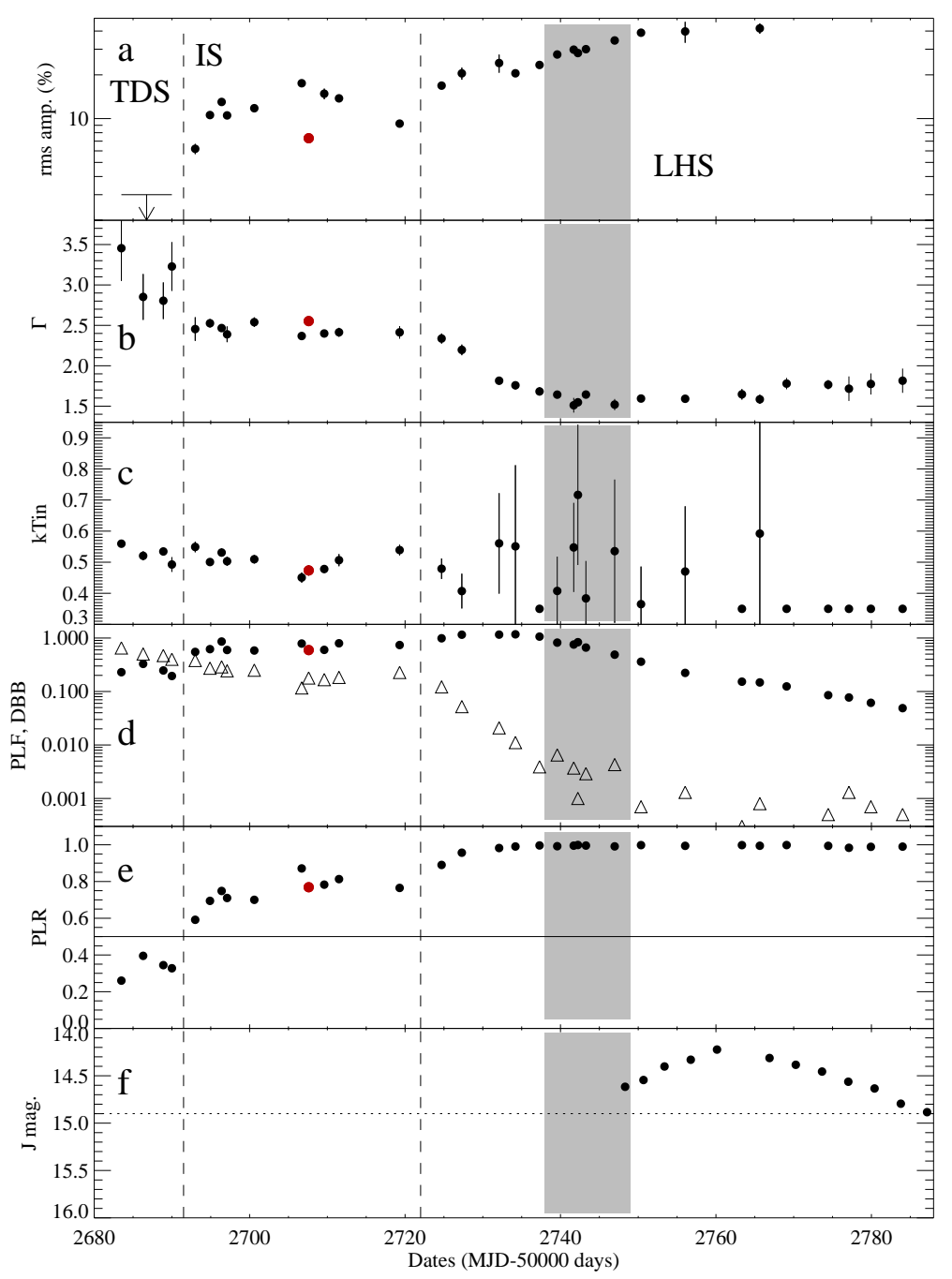

Figure 4: GX 339-4; the evolution of (a) the total rms amplitude of variability in 3-30 keV band, (b) the photon index $(\Gamma),(\mathrm{c})$ the inner disk temperature $\mathrm{k} T_{\text {in }}(\mathrm{d})$ unabsorbed power-law flux and the disk blackbody flux in 3-25 keV band in units of $10^{-9} \mathrm{ergs} \mathrm{cm}^{-2} \mathrm{~s}^{-1}$, (e) the PLR (f) J magnitudes, the dotted line is the background level. The dashed lines indicate approximate times of state transitions. The gray area shows the observations during which a high energy cut-off is required in the fit. The solid line in (e) indicates threshold to observe variability. The observation shown in red has B type QPO.

source was observed intensely in many wavelengths (see tahti.mit.edu/opensource/1655 for all references regarding multi-wavelength coverage, and also Migliari et al. in this proceedings). We analyzed the public data from MJD 53625 to MJD 53645, covering the state transitions during the decay. The ridge emission was determined using quiescent state observations at the end of the outburst.

Fig. 5 shows the evolution during the decay. The fast and slow transitions are again observed. A larger region requires a cut-off in the spectral fits, and the cut-off disappears after the spectrum becomes hardest. The monotonously decaying QPO frequencies are also shown. 


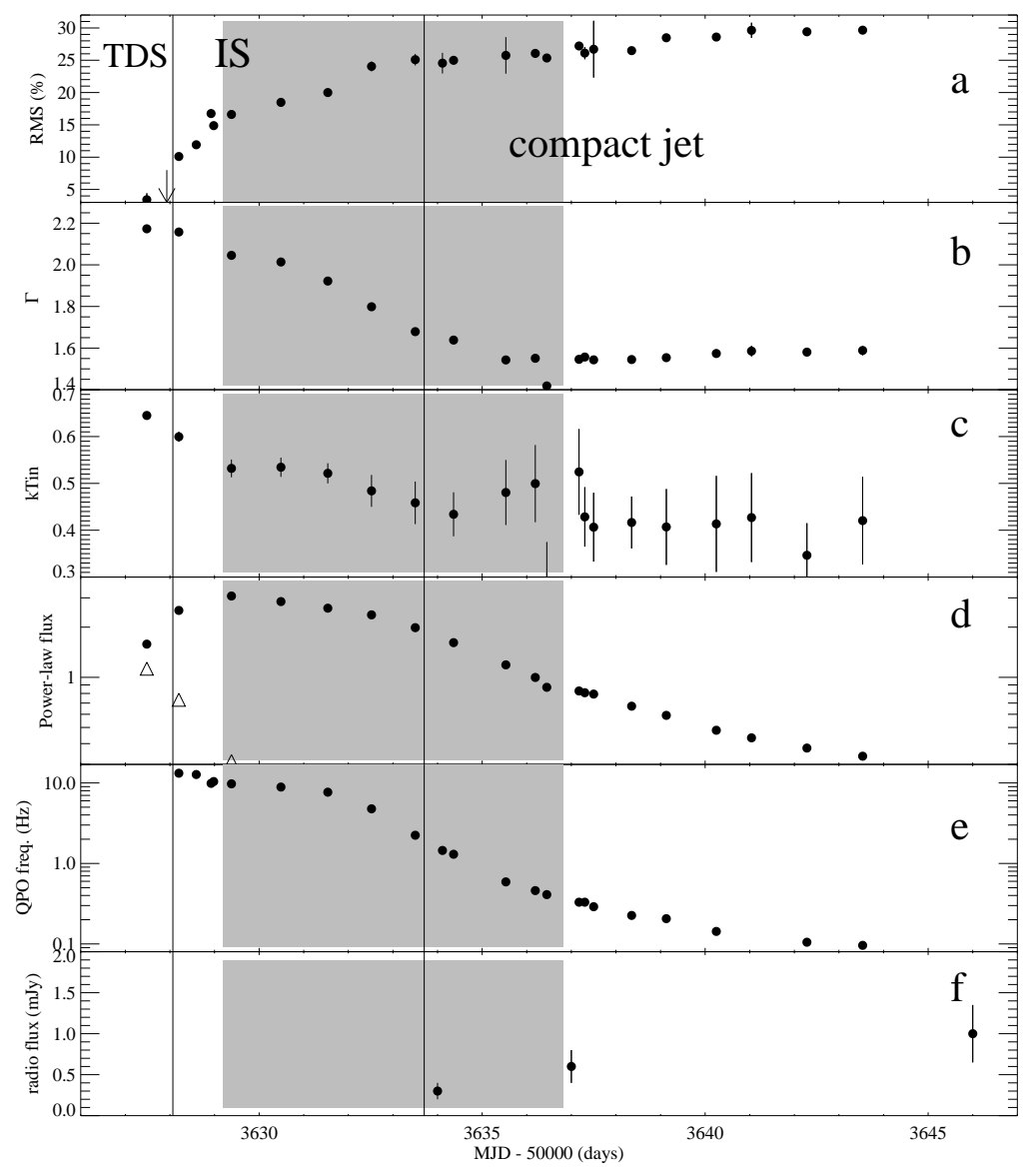

Figure 5: GRO J1655-40; the evolution of (a) the total rms amplitude of variability in 3-30 keV band, (b) the photon index $(\Gamma)$, (c) the inner disk temperature $\mathrm{k} T_{\text {in }}$ (d) unabsorbed power-law flux and the disk blackbody flux in 3-25 keV band in units of $10^{-9} \mathrm{ergs} \mathrm{cm}^{-2} \mathrm{~s}^{-1}$, (e) the QPO frequency, (f) radio flux in mJy. The first solid line indicates the time of transition from the TDS to the IS, the second solid line indicates the time that the source is detected in radio for the first time. The gray area shows the observations during which a high energy cut-off is required in the fit.

\section{Summary of results}

Based on the evolution of the four sources discussed in this work, and also from other work $[17,15]$, we summarize our results as follows (see also Fig. 6):

- A fast transition from the TDS to the hard intermediate state occurs within a timescale of 1 day. The transition can easily be seen in the PSD, and the increase in the rms amplitude of variability is accompanied by an increase in the power-law flux. The spectral index does not change much during this transition. No high energy cut-off is required around this transition.

- Five to seven days later the spectral index begins to drop and within seven to 10 days, the source reaches its hardest state. While the spectrum hardens, most sources show a cut-off in the spectral index. 


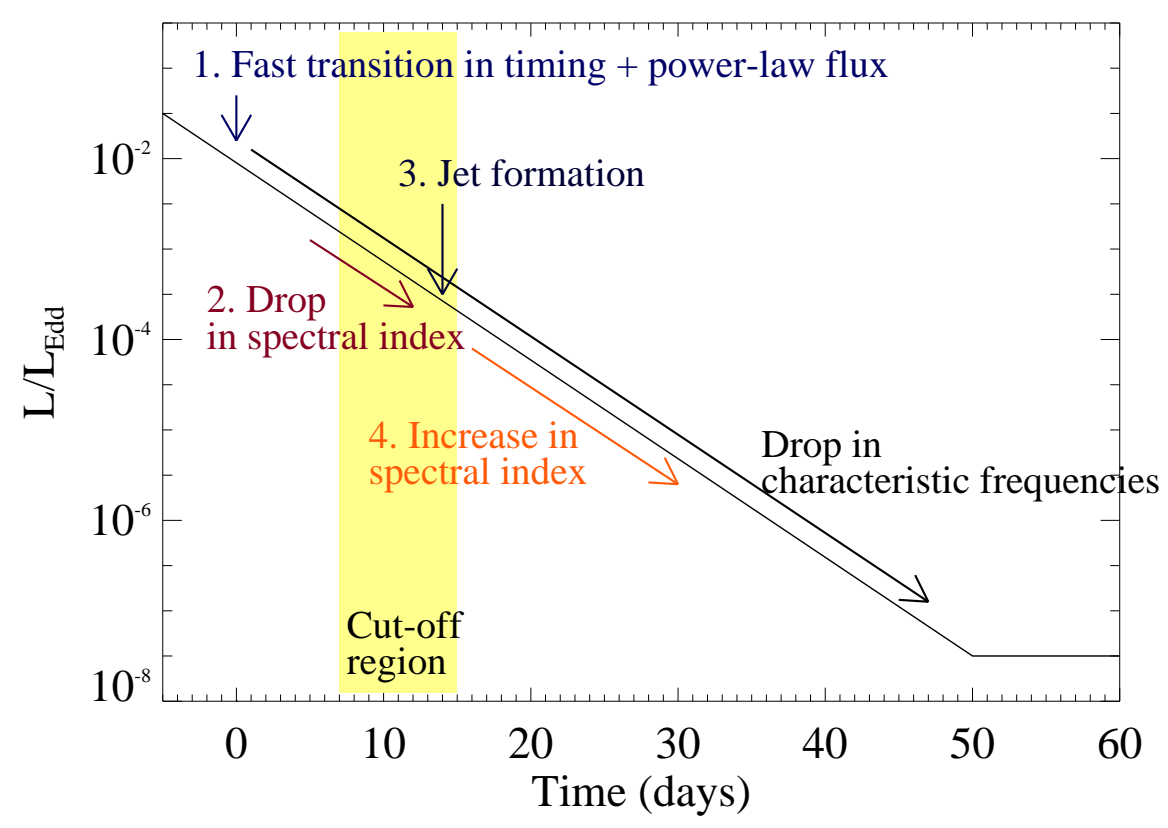

Figure 6: Brief summary of the evolution. Time 0 is the date of transition from the TDS to the hard intermediate state (the fast transition). The timescales and luminosities are approximate and vary from source to source.

- Close to the hardest spectrum, emission from the compact jet is observed in radio or OIR. The cut-off disappears at most two to three days after the first sign of the compact jet.

- The formation of the jet needs PLR $>0.99$ and spectral index $<1.7$ (however, see Discussion for XTE J1720-318.

- Some sources show an increase in spectral index after the jet is observed.

- During the entire decay, the characteristic frequencies (QPO or peak frequencies of Lorentzians) decrease monotonously.

\section{Discussion}

The fast and slow transitions happen for all sources. However, there are a few exceptions that need to be discussed for the other items listed above. Not all sources require a cut-off in their spectral fits, e.g. H 1743-322. For sources discussed in this work, to be able to detect the jet in the radio or OIR, PLR $>0.99$ and spectral index $<1.7$ are necessary conditions. However, for XTE J1720-318, there is a radio detection during the decay while the source is still soft (PLR $\sim 0.85$, and spectral index $\sim 2$ ). Though, it is not clear whether this radio detection is from a compact jet, as it was observed in one frequency (see proceeding by S. Chaty). It is not clear that all sources show a softening at the end of the outbursts. A study by Corbel et al. [7] indicates that low orbital period sources tend to soften while approaching quiescence. The sources that 
show softening are XTE J1550-564, 4U 1543-47, XTE J1118+480, A0620-00, GX 339-4, and XTE J1650-500. For 4U 1543-47, and GX 339-4, the softening starts with the onset of radio and OIR activity.

In the context of the existing models we try to understand the evolution observed in these sources. The fast transition is seen as a sharp rise in rms amplitudes accompanied with a rise in power-law flux. The "flip-flop" in H 1743-322, and the requirement of a PLR limit to observe variability all indicate that the timing signatures are only present for a portion of the power-law flux. There may be two distinct physical mechanisms creating the power-law flux, one with timing signature, the other not. In this case these would have to conspire to give one spectral index. We suggested that there is only one process, the Compton scattering, and the fast transition is due to the emergence of an additional soft seed photon component, such as synchrotron radiation [19]. The slow transition on the other hand simply seems to be an increase in coronal temperature, producing harder spectra.

One of the most important results of this work is establishing the onset of jet emission in radio or OIR. The jet is observed close to the end of the second transition, while the spectrum is hardest. Therefore the launch of the jet does not cause state transitions, but is a consequence of the evolution of X-ray spectral properties. Some jet launching models require the formation of a geometrically thick disk (corona) first [21], and our results are consistent with this.

The presence or lack of high energy cut-offs has become an important parameter in understanding GBHTs as they seem to be closely related to state transitions [18, 2]. Except for H 1743-322 which required no cut-off at all, the cut-off seems to disappear once the jet is observed in radio or OIR. The formation of the jet may have been injecting non-thermal electrons into the corona, thereby creating a hard tail [4]. Alternatively, the coronal temperature may become too high to detect the cut-off with HEXTE, especially for low fluxes.

Finally, some sources show a softening after the initial detection of the jet while the source is still radio bright. The softening may simply be due to lack of high temperature electrons in a Comptonizing corona. It may also be a direct consequence of the jet in X-ray spectrum [31].

\section{Acknowledgments}

E.K. is supported by the European Commission through a FP6 Marie-Curie International Reintegration Grant (INDAM). E.K. acknowledges partial support of TÜBİTAK. This project is supported by NASA Grant NNG06GB90G. EK thanks Rob Fender, Catherine Brocksopp, and Micheal Rupen for radio data, Michelle Buxton and Charles Bailyn for optical \& infrared data. EK thanks the organizers of the VIth Microquasar Workshop, and Sara Markoff, Piergiorgio Casella, and Jeroen Homan for useful discussions during the meeting. R.E.R and K.P acknowledge NASA support NAS5-30720.

\section{References}

[1] Belloni, T., 2005, in AIP Conf. Proc. 797, 197, astro-ph/0504185

[2] Belloni, T., et al., 2006, MNRAS, 367, 1113

[3] Berger, M., \& van der Klis, M., 1994, A\&A, 292, 175 
[4] Coppi, P. S., 1999, in ASP Conf. Ser. 161: High Energy Processes in Accreting Black Hole, eds. Poutanen, J. and Svensson, R.

[5] Corbel, S., et al, 2000, A\&A, 359, 251

[6] Corbel, S., \& Fender, R. P., 2002, ApJ, 573, L35

[7] Corbel, S., Tomsick, J. A., Kaaret, P., 2006, ApJ, 636, 971

[8] Ebisawa, K., et al., 1994, PASJ, 46, 375

[9] Esin, A. A., McClintock, J. E., \& Narayan, R., 1997, ApJ, 489, 865

[10] Fender, R. P., 2001, MNRAS, 322, 31

[11] Fender, R. P., \& Kuulkers, E., 2001, MNRAS, 324, 923

[12] Homan, J., et al., 2001, ApJS, 132, 377

[13] Homan, J., \& Belloni, T., 2005, ApSS, 300, 107

[14] Kalemci, E., et al., 2001, ApJ, 563, 239

[15] Kalemci, E., 2002, Ph.D. Thesis, University of California, San Diego

[16] Kalemci, E., et al., 2003, ApJ, 586, 419

[17] Kalemci, E., et al., 2004, ApJ, 603, 231

[18] Kalemci, E., et al., 2005, ApJ, 622, 508

[19] Kalemci, E., et al., 2006, ApJ, 639, 340

[20] McClintock, J. E., \& Remillard, R. A., 2006, ARA\&A, 44, 49, astro-ph/0606352

[21] Meier, D. L., Koide, S., \& Uchida, Y., 2001, Science, 291, 84

[22] Miyamoto, S., \& Kitamoto, S., 1989, Nature, 342, 773

[23] Pottschmidt, K., 2002, PhD Thesis, Univ. of Tübingen

[24] Revnivtsev, M., 2003, A\&A, 410, 865

[25] Russell, D. M. et al., 2006, MNRAS, 371, 1334

[26] Tomsick, J. A., \& Kaaret, P., 2000, ApJ, 537, 448

[27] Tomsick, J. A., Corbel, S., \& Kaaret, P., 2001, ApJ, 563, 229

[28] Tomsick, J. A., \& Kalemci, E., 2003, ATEL, 198

[29] Tomsick, J. A., Kalemci, E., Corbel, S., \& Kaaret, P., 2003, ApJ, 592, 1100

[30] Valinia, A., \& Marshal, F. E., 1998, ApJ, 505, 134

[31] Yuan, F., Cui, W., Narayan, R., 2005, ApJ, 620, 905

[32] Zdziarski, A. A., Poutanen, J., Paciesas, W. S., \& Wen, L., 2002, ApJ, 578, 357

[33] Zhang, W., et al., 1995, ApJ, 449, 930 